\title{
Real Zeuthen Numbers for Two Lines
}

\section{Benoit Bertrand}

Section de Mathématiques, Université de Genève, Case postale 64, 1211 Genève 4, Suisse

Correspondence to be sent to: benoit.bertrand@math.unige.ch

Given three natural numbers $k, l, d$ such that $k+l=d(d+3) / 2$, the Zeuthen number $N_{d}(l)$ is the number of nonsingular complex algebraic curves of degree $d$ passing through $k$ points and tangent to $l$ lines in $\mathbb{P}^{2}$. It does not depend on the generic configuration $C$ of points and lines chosen. If the points and lines are real, the corresponding number $N_{d}^{\mathbb{R}}(l, C)$ of real curves usually depends on the configuration chosen. We use Mikhalkin's tropical correspondence theorem to prove that for two lines, the real Zeuthen problem is maximal: there exists a configuration $C$ such that $N_{d}^{\mathbb{R}}(2, C)=N_{d}(2)$. The correspondence theorem reduces the computation to counting certain lattice paths with multiplicities.

\section{Introduction}

Given $l$ lines and $k=d(d+3) / 2-l$ points in $\mathbb{C} P^{2}$, how many nonsingular complex algebraic curves of degree $d$ pass through the $k$ points and are tangent to the $l$ lines? This is a particular instance of the Zeuthen problem. For generic configurations of points and lines, there are finitely many solutions to the problem and we call Zeuthen number the number $N_{d}(l)$ of solutions. Here we consider the corresponding question for real data: assume that the points and the lines are real, how many degree $d$ real curves pass

This work is dedicated to the memory of Felice Ronga.

Received November 9, 2007; Revised November 9, 2007; Accepted February 1, 2008

Communicated by Prof. Andrei Zelevinsky

(C) The Author 2008. Published by Oxford University Press. All rights reserved. For permissions, please e-mail: journals.permissions@oxfordjournals.org. 
through the $k$ points and are tangent to the $l$ lines? In other words, what values can take the real Zeuthen number $N_{d}^{\mathbb{R}}(l, C)$ of real solutions? This number usually depends on the configuration $C$, and, clearly, the (invariant) number of complex solutions is an upper bound.

Whether there exists a generic configuration for which all the solutions are real is a natural and classical question in real enumerative geometry. It is said that the problem is maximal if such a configuration exists. For $l=1$, it was shown by Ronga [4] that the Zeuthen problem is maximal in the above sense (i.e. all the curves can be real). In this article, we show that the problem for two lines is also maximal.

Theorem 1.1. For any integer $d \geq 2$, there exists a configuration $C$ of two real lines and $d(d+3) / 2-2$ real points such that all the degree $d$ curves passing through the points and tangent to the lines are real,

$$
N_{d}^{\mathbb{R}}(2, C)=N_{d}(2)
$$

The techniques we use are those developed by Mikhalkin in [3]. The statement is proved using correspondence theorems to tropicalize the problem and the lattice path algorithm to count the number of tropical curves. I would like to thank G. Mikhalkin and E. Shustin for useful discussions.

\section{Tropical Tangency and Algorithm}

\subsection{Tropical tangency}

We use here the terminology introduced in [3] without recalling definitions and basic properties of tropical curves. General tropical tangency has been discussed in Mikhalkin's note [2]. Here we consider a simple special case of tangency with respect to toric divisors i.e. in our projective plane setting, the three axes which are the closures of one-dimensional orbits of the torus action (see also [1]). Recall that a plane tropical curve is dual to a subdivision of its Newton polygon such that the weight of an edge of the curve is equal to lattice length of its dual edge. A simple tangency to a coordinate axis in the algebraic world translates to a weight 2 unbounded edge in tropical world.

Definition 2.1. We say that a tropical curve $C$ with Newton polygon $\Delta$ is tangent to an axis corresponding to the edge $\delta \subset \Delta$ if in the dual subdivision there is a 1-simplex of length greater or equal to 2 included in $\delta$ (see Figure 1.). 

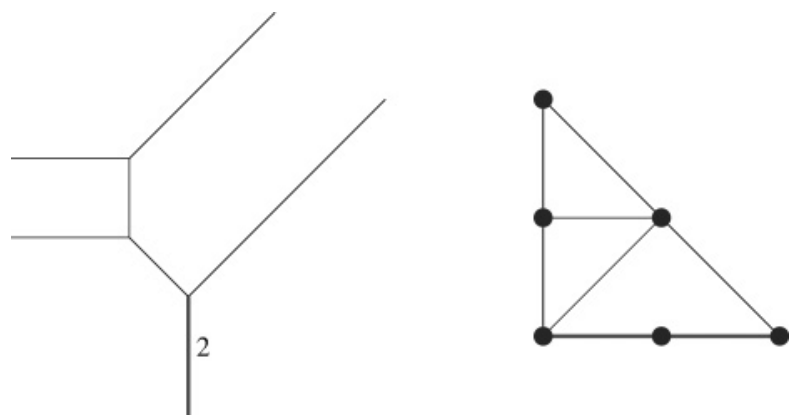

Fig. 1.. Tropical conic tangent to the horizontal axis and dual triangulation of its Newton polygon

We only consider here simple tangencies (i.e. the weight of the corresponding edge is 2).

Under the degeneration process described in [3] Section 6.1, a family of curves tangent to some axes and passing through the right number of points has a tropical limit curve which is tangent (in the above sense) to the axes.

All configurations considered here will be generic. The multiplicity $\operatorname{mult}(A)$ of a tropical curve $A$ and the multiplicity $\operatorname{mult}_{\mathbb{R}}(A)$ of a real tropical curve is defined as in [3], Definitions 4.15 and 7.10. The proof of Theorem 1.1 we give will use the so-called lattice path algorithm of counting tropical curves with multiplicity. The multiplicity of a path is explained in Section 2.2.

Theorem 2.2. Let $l \leq 3$ be a positive integer. The number of complex algebraic curves of degree $d$ passing through $d(d+3) / 2-l$ points and tangent to $l$ lines is equal to the number of degree $d$ tropical curves through $d(d+3) / 2-l$ points and tangent to $l$ chosen axes counted with multiplicities.

Theorem 2.3. Let $l \leq 3$ be a positive integer. Let $T$ be a configuration of $d(d+3) / 2-l$ points. Let $N_{\text {trop,d,T }}^{\mathbb{R}}$ be the number of degree $d$ real tropical curves counted with multiplicities passing through $C$ and tangent to $l$ chosen axes. Then there exists a configuration $C$ of $d(d+3) / 2-l$ real points and $l$ real lines, such that $N_{d}^{\mathbb{R}}(l, C)=N_{\text {trop, },,^{T}}^{\mathbb{R}}$.

The first statement can be extracted from [2] and [3] and the second one can be deduced from the first one analyzing, as in [3], which tropical curves actually produce real algebraic curves (see [3] Section 8, p. 365 and Propositions 6.17, 6.18, 8.21, and Theorems 1 
and 3). Alternatively, one can also deduce Theorem 2.2 from Shustin's Lemma 5.4 and Theorem 5 in [5].

\subsection{Lattice path algorithms}

As in [3] Sections 7.2 and 7.4, placing the points on a line of negative irrational slope and sufficiently far away from one another, one obtains algorithms to count the relevant tropical curves. We describe below in our special cases the slightly modified Mikhalkin's algorithms (see [3] Sections 7.2 and 7.4) that take tangencies into account. We refer to [3] for a more developed presentation of lattice path algorithms.

Let $\Delta$ be the triangle with vertices $(0,0),(d, 0),(0, d)$ (that is the Newton polygon of a generic curve of degree $d$ ). Let $l \leq 3$ be a positive integer and $p_{1}, \ldots, p_{l}$ be integer points on $l$ different edges of $\Delta$. Choose a nearly horizontal line $L$ of $\mathbb{R}^{2}$ with a very small negative irrational slope and consider the orthogonal projection $\lambda: \mathbb{R}^{2} \rightarrow L \sim \mathbb{R}$. Let $p$ (resp. $q$ ) be the point of $\Delta$ where $\lambda$ reaches it's minimum (resp. maximum). Let $\gamma:[0, n] \rightarrow \Delta$ be an increasing (piecewise linear) lattice path from $p$ to $q$ avoiding the $p_{i}$ 's (i.e. $\gamma([0, n] \cap \mathbb{Z}) \subset \Delta \cap \mathbb{Z}^{2} \backslash\left\{p_{1}, \ldots, p_{l}\right\}$ and $\lambda \circ \gamma$ is increasing). We define inductively positive and negative multiplicities $\mu_{ \pm}$of $\gamma$. Let $\alpha_{+}$(resp. $\alpha_{-}$) be the path supported by the upper edge of $\Delta$ (resp. the vertical and lower edges of $\Delta$ ). The multiplicities $\mu_{ \pm}\left(\alpha_{ \pm}\right)$ of $\alpha_{ \pm}$are 1 . If the path $\gamma$ is neither $\alpha_{+}$nor $\alpha_{-}$then it divides $\Delta$ into two closed regions $\Delta_{+}$and $\Delta_{-}$, where $\Delta_{ \pm}$contains $\alpha_{ \pm}$. Let $k$ be the smallest integer such that $\Delta_{ \pm}$is locally strictly convex at $\gamma(k)$. Consider the path $\gamma^{\prime}:[0, n-1] \rightarrow \Delta$ defined by $\gamma^{\prime}(j)=\gamma(j)$ if $j<k$ and $\gamma^{\prime}(j)=\gamma(j+1)$ if $j \geq k$. We set

$$
\mu_{ \pm}(\gamma):=2 \operatorname{area}(T) \mu_{ \pm}\left(\gamma^{\prime}\right)
$$

where $T$ is the triangle with vertices $\gamma(k-1), \gamma(k), \gamma(k+1)$. The multiplicity $\mu(\gamma)$ is defined by $\mu(\gamma)=\mu_{+}(\gamma) \cdot \mu_{-}(\gamma)$. Here is the statement in our particular case.

Let $\delta_{1}, \ldots, \delta_{3}$ be edges of $\Delta$ and $\eta_{i}$ be the set of integer points in the relative interior of $\delta_{i}$.

Theorem 2.4. Let $l \leq 3$ be a positive integer. The number $N_{d}(l)$ is equal to the sum of the multiplicities of the $\lambda$-increasing lattice paths $[0, d(d+3) / 2-l] \rightarrow \Delta$ avoiding $p_{1}, \ldots, p_{l}$ over all $\left\{p_{1}, \ldots, p_{l}\right\} \in \eta_{1} \times \cdots \times \eta_{l}$. 
There is a real version of the algorithm to count real curves. We briefly explain how to obtain it from the previous one in our special case and refer to [3] for details.

One now needs to consider "signs" on intervals $[j-1, j]$ for $j=1, \ldots, n$ and "phases" of edges which are the images of those intervals. Let $s \in \mathbb{Z}_{2}^{2}$ be a choice of "sign" on each interval. Suppose $\gamma(j)-\gamma(j-1)=\left(y_{j}, x_{j}\right) \in \mathbb{Z}^{2}, j=1, \ldots, n$. Let $S_{j}$ be the quotient of $\mathbb{Z}_{2}^{2}$ by the equivalence relation $(X, Y) \sim\left(X+X_{i} \bmod 2, Y+y_{i} \bmod 2\right),(X, Y) \in \mathbb{Z}_{2}^{2}$. It induces a phase $\sigma_{j}(s) \in S_{j}$ on each corresponding edge $[\gamma(j-1), \gamma(j)]$ of the image of $\gamma$ in $\Delta$. If $S=\left(S_{1}, \ldots, S_{n}\right) \in\left(\mathbb{Z}_{2}^{2}\right)^{n}$ is a choice of "signs," we denote by $\sigma(S)$ the $n$-tuple of induced phases $\sigma_{1}\left(S_{1}\right), \ldots, \sigma_{n}\left(S_{n}\right)$.

Let $\sigma=\left(\sigma_{j}\right)_{j=1, \ldots, n}, \sigma_{j} \in S_{j}$ be any choice of phases on the edges of the image of $\gamma$. The real multiplicity $\mu^{\mathbb{R}}(\gamma, \sigma)$ of a path $\gamma$ equipped with phases $\sigma$ is given by $\mu^{\mathbb{R}}(\gamma, \sigma)=\mu_{+}^{\mathbb{R}}(\gamma, \sigma) \cdot \mu_{-}^{\mathbb{R}}(\gamma, \sigma)$, where $\mu_{ \pm}^{\mathbb{R}}(\gamma, \sigma)$ are defined inductively as above. The path $\gamma^{\prime}$ is obtained as before and we set $\mu_{ \pm}^{\mathbb{R}}\left(\alpha_{ \pm}, \sigma\right)=1$ and

$$
\mu_{ \pm}^{\mathbb{R}}(\gamma, \sigma)=a(T) \mu_{ \pm}^{\mathbb{R}}\left(\gamma^{\prime}, \sigma^{\prime}\right)
$$

where

- $a(T)=1$ if the lattice area (twice the usual one) of $T$ is odd. In this case the phase $\sigma_{k}^{\prime}$ is chosen such that the three classes $\sigma_{k}, \sigma_{k+1}$, and $\sigma_{k}^{\prime}$ do not share a common element.

- $a(T)=0$ if all sides of $T$ have even lattice length and $\sigma_{k} \neq \sigma_{k+1}$.

- $a(T)=4$ if all sides of $T$ have even lattice length and $\sigma_{k}=\sigma_{k+1}$. Then define $\sigma_{k}^{\prime}:=\sigma_{k}$.

- $a(T)=0$ if $\sigma_{k}$ and $\sigma_{k+1}$ do not have a common element.

- $a(T)=2$ if $T$ has exactly one even side distinct from $[\gamma(k-1), \gamma(k+1)]$. Then $\sigma_{k}^{\prime}$ is defined by the fact that it should have a common element with $\sigma_{k}$ and $\sigma_{k+1}$.

- If $[\gamma(k-1), \gamma(k+1)]$ is the only even side of $T$, then one should consider the path $\gamma^{\prime}$ with the two choices of $\sigma_{k}^{\prime}$ satisfying the above condition (i.e. $\mu_{ \pm}^{\mathbb{R}}(\gamma, \sigma)=$ $\left.\mu_{ \pm}^{\mathbb{R}}\left(\gamma^{\prime}, \sigma_{1}^{\prime}\right)+\mu_{ \pm}^{\mathbb{R}}\left(\gamma^{\prime}, \sigma_{2}^{\prime}\right)\right)$.

Theorem 2.5. Let $l \leq 3$ be a positive integer. For any choice of $S \in\left(\mathbb{Z}_{2}^{2}\right)^{d(d+3) / 2-l}$, there exists a configuration of $d(d+3) / 2-l$ generic points such that the number of degree $d$ real curves among the $N_{d}(l)$ complex ones is equal to the sum of the multiplicities $\mu^{\mathbb{R}}(\gamma, \sigma(S))$ of 


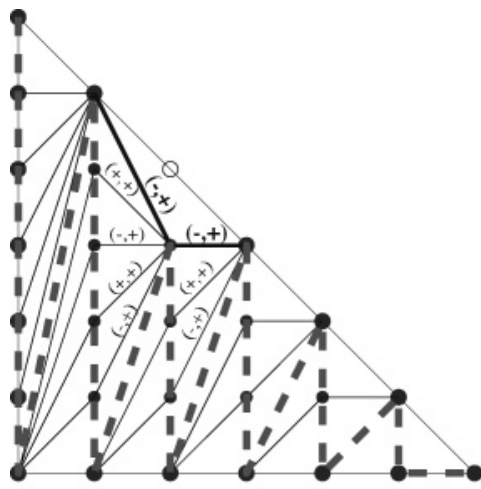

Tangency to the line at infinity.

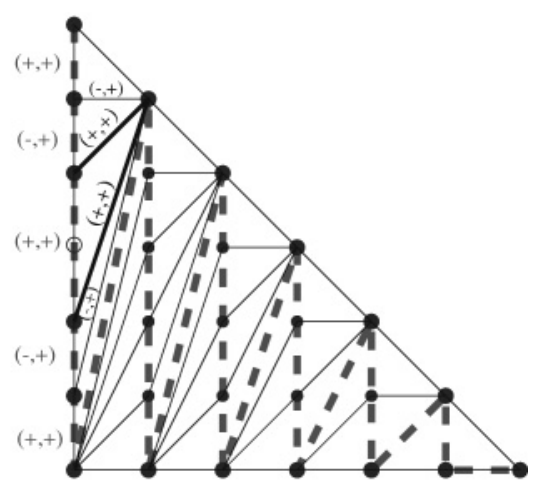

Tangency to the vertical axis.

Fig. 2.. $\quad$ Case of one line

the $\lambda$-increasing signed lattice paths $(\gamma, \sigma(S)):[0, d(d+3) / 2-l] \rightarrow \Delta$ avoiding $p_{1}, \ldots, p_{l}$ over all $\left\{p_{1}, \ldots, p_{l}\right\} \in \delta_{1} \times \cdots \times \delta_{l}$.

\section{Maximality}

\subsection{Case of one line}

The case of one line was studied by Felice Ronga [4] who proved maximality and completeness of the real Zeuthen problem. We give a tropical proof of the maximality for one line.

As we can choose the line to be any of the axes, we will consider two cases: first, the line at infinity and then ordinate axis. To count the lines tangent to the infinity line, we need to count lattice paths of maximal length avoiding one of the interior points $(m, d-$ $m$ ) of the hypothenus $h$ of $\Delta$. Consider the constant sign sequence $((+,+), \ldots,(+,+))$ (we denote by + the zero element of $\mathbb{Z}_{2}$ and by - the nonzero one). We have $d-1$ choices for the point $(m, d-m)$ and, as shown in Figure 2., for each one the lattice path has multiplicity 2. Thus, the total number of curves passing through our configuration is $2(d-1)$. When considering the ordinate axis, the paths must avoid a point on the vertical edge $v$ of $\Delta$ and we choose the sign sequence $S_{d(d+3) / 2-1}$, which is constant and equal to $(+,+)$ except for the $d-1$ first terms that are $(-,+),(+,+),(-,+),(+,+), \ldots,(-,+),(+,+)$ for $d$ odd and $(+,+),(-,+),(+,+), \ldots,(-,+),(+,+)$ for $d$ even. Again as shown in Figure 2., each path has multiplicity 2 . 


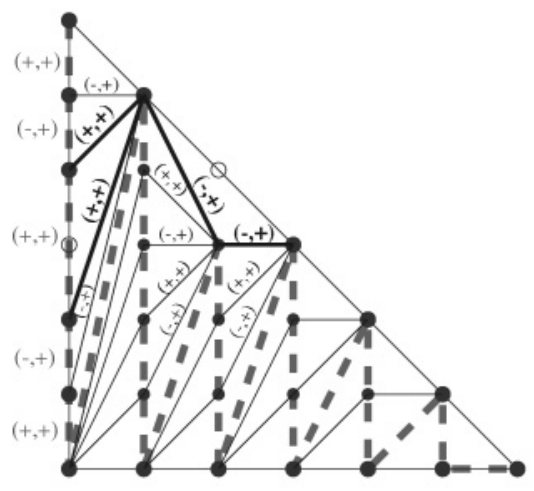

Splitting case.

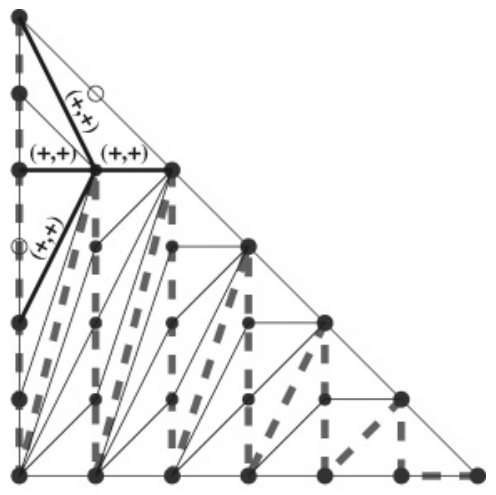

Non independent case.

Fig. 3.. The two-lines case

\subsection{Main theorem}

We prove that for two lines, there is a configuration of $d(d+3) / 2-2$ points and two lines for which all the (nonsingular) curves of degree $d$ passing through the points and tangent to the lines are real.

Proof. We can choose the lines to be the infinity line and the ordinate axis. Consider the sign sequence $S_{d(d+3) / 2-2}$ of length $d(d+3) / 2-2$ defined in Section 3.1. We just need to see that for each pair of integer points lying respectively in the interior of the edges $h$ and $v$, we have a lattice path of multiplicity 4 to prove that $N_{d}^{\mathbb{R}}(2, C)=(2(d-1))^{2}$.

If the point on $h$ is not $(1, d-1)$, then the picture splits into two independent parts: the band $[0,1] \times[0, d]$ and the triangle with vertices $(0,1),(0, d)$, and $(d-1,1)$. Both parts were studied for the case $l=1$ in previous section and contribute each for 2 in the multiplicity (see Figure 3.).

If the first point is $(1, d-1)$, one just needs to notice that the signed segments on $v$ behave exactly as if the one of length 2 was split in two of length 1 and the sign distribution was that of second case of Section 3.1. Hence, the multiplicity of these paths is also 4 (see Figure 3.).

\section{Acknowledgments}

The author was partially supported by the European research network IHP-RAAG contract HPRNCT-2001-00271 and part of the work was done at Max Planck Institute for Mathematics in Bonn 
8 B. Bertrand

\section{References}

[1] Gathmann, A., and H. Markwig. "The Caporaso-Harris formula and plane relative GromovWitten invariants in tropical geometry." Mathematische Annalen 338, no. 4 (2007): 845-68.

[2] Mikhalkin, G. "Tropical computation of Zeuthen's numbers of toric surfaces." http://www.math.toronto.edu/mikha/XN.ps. (accessed February 18, 2008).

[3] Mikhalkin, G. "Enumerative tropical algebraic geometry in $\mathbb{R}^{2}$." Journal of the American Mathematical Society 18, no. 2 (2005): 313-77 (electronic).

[4] Ronga, F. "A real Riemann-Hurwitz theorem." Boletim da Sociedade Brasileira de Matim ática, Nova Série 31, no. 2 (2000): 175-87.

[5] Shustin, E. "A tropical approach to enumerative geometry." Algebra i Analiz 17, no. 2 (2005): $170-214$. 